\title{
Membrane Triangles with Drilling Degrees of Freedom
}

P. Fajman

An accurate triangular plane element with drilling degrees of freedom is shown in this paper. This element can be successfully used for solving linear and nonlinear problems. The main advantage of this element is that the stiffness matrix is obtained from pure deformations elongations of the edges. This aproach is very suitable for nonlinear analysis, where the unbalanced forces can be obtained directly from elongations of edges.

Keywords: triangular, element, elongations, rigid, displacement.

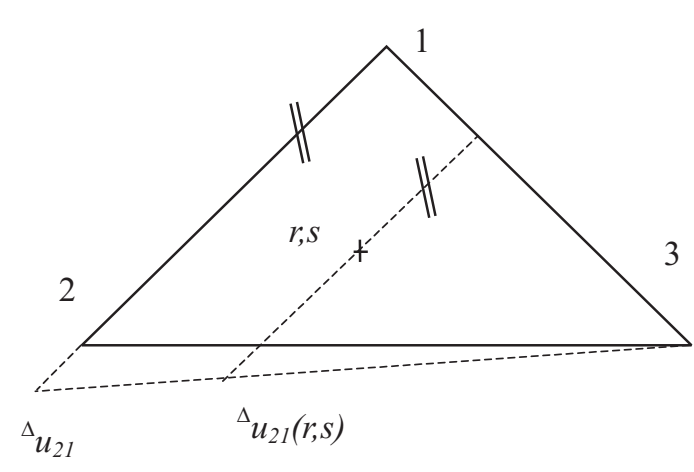

Fig. 1: Node displacement field ${ }^{\Delta} u_{21}(r, s)$

\section{Introduction}

The basic unknowns are elongations in the directions of edges $\Delta_{21}, \Delta_{32}, \Delta_{13}$ and pure rotations $\varphi_{i z}$ of a small neighbourhood of a node (considered rigid) about the $z$-axis passing through vertex $i$, where $i=1,2,3$. The effect of pure swing is transferred to the elongations of the edges and is taken into account in formulating of the element stiffness matrix. The static matrix (see section "Element Stiffness Matrix") supports the rigid body motion (RBM).

\section{Element displacement}

Derivation of the element stiffness matrix relies on Hrenikoff's idea that a triangular element can be represented by a system of lines parallel to the edges. Owing to this idea we further assume that displacements along individual element sides are mutually independent. Triangular coordinates $L_{i}$

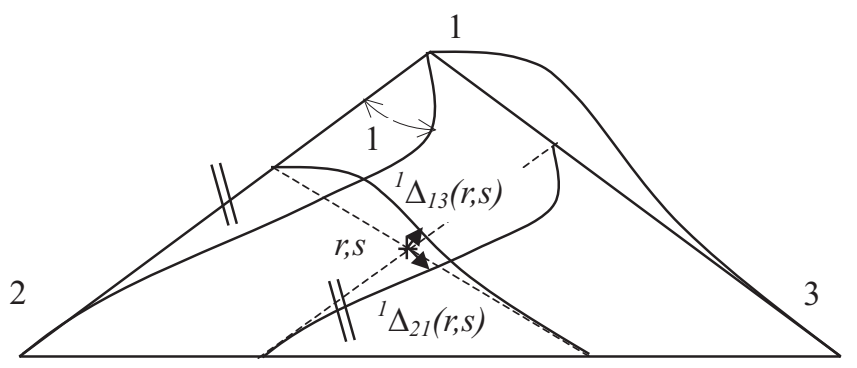

Fig. 2: Extension from pure rotation $\varphi_{1}=1$

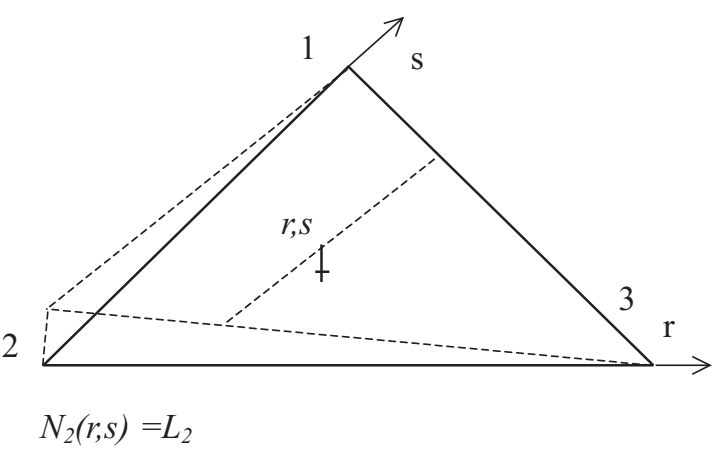

(appendix A) are used to describe the basic displacement field $u_{i j}(r, s)$ derived from pure deformations and rotations. Here, the pure deformations are defined through elongations of the individual edges and denoted as $u_{21}, u_{32}, \ldots, u_{13}$. They are composed of two parts - pure extensions due to node displacements and pure extensions caused by node rotations.

Pure extensions in terms of nodal elongations. With the help of these quantities we may express the elongations of edges ${ }^{\Delta} u_{i j}(r, s)$ at the point $r, s$ by

$$
{ }^{\Delta} u_{i j}(r, s)=N_{i}(r, s) \cdot{ }^{\Delta} u_{i j}=L_{i} \cdot{ }^{\Delta} u_{i j} .
$$

Pure extensions in terms of nodal rotations. Associating the rotation in node 1 with $\varphi_{1}=1$ we write the pure extension along the edges in terms of cubic shape functions in Fig. 2.

$$
\begin{aligned}
& { }^{k} \Delta_{21}(r, s)=L_{2} L_{1}^{2} l_{21} \varphi_{1}, \\
& { }^{k} \Delta_{13}(r, s)=L_{3} L_{1}^{2} l_{13} \varphi_{1},
\end{aligned}
$$

where $l_{i j}$ are the lengths associated with sides $i j$.

Rotation $\varphi_{i}$ causes in node $(r, s)$ two displacements ${ }^{k} \Delta_{i j}$ perpendicular to lines that are parallel with sides $i j$ (Fig. 2). Superscript $k$ means the node about which the rotation takes place. Extensions ${ }^{\varphi i} u_{i j}(r, s)$ of three lines parallel with edges, which pass point $(r, s)$, due to the vertex rotations $\varphi_{i}$ (superscript $\varphi_{i}$ ) need to be expressed. These elongations $\varphi i u_{i j}(r, s)$ are obtained by projecting ${ }^{k} \Delta_{i j}$ in the corresponding directions $i j$.

Elongations from the rotation in node 1 (Fig. 3) can be written as 


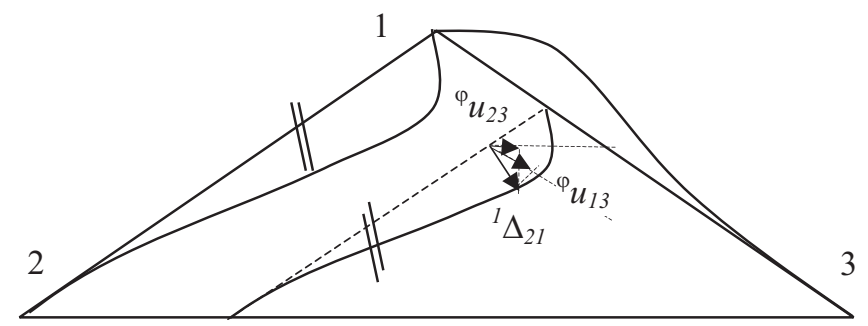

Fig. 3: Relation between node rotation 1 and elongations

$$
\begin{aligned}
{ }^{\varphi 1} u_{i j}(r, s) & ={ }^{1} \Delta_{21} \mathbf{n}_{21} \mathbf{t}_{i j}+{ }^{1} \Delta_{13} \mathbf{n}_{13} \mathbf{t}_{i j}= \\
& =-\varphi_{1} l_{21} L_{2} L_{1}^{2} \mathbf{n}_{21} \mathbf{t}_{i j}+\varphi_{1} l_{13} L_{3} L_{1}^{2} \mathbf{n}_{13} \mathbf{t}_{i j},
\end{aligned}
$$

where $i=2,3,1$ and $j=1,2,3$,

and

$\left\{\mathbf{n}_{i j}\right\}=\left\{n_{x i j}, n_{y i j}\right\}=\left\{\cos \alpha_{n i j}, \sin \alpha_{n i j}\right\}$ is the outward unit normal vector of the element side $i j$,

$\left\{\mathbf{t}_{i j}\right\}=\left\{t_{x i j}, t_{y i j}\right\}=\left\{\cos \alpha_{n i j}, \sin \alpha_{n i j}\right\}$ is the unit vector of the element side $i j$ (Fig. 4).

The other contributions from the rotations in nodes 2 and 3 are obtained by cyclic index replacement. Elongation ${ }^{\varphi}{ }^{i} u_{i j}(r, s)$ from three node rotations are obtained by summation of the separate contributions ${ }^{\varphi 1} u_{i j}(r, s),{ }^{\varphi 2} u_{i j}(r, s)$, ${ }^{3} u_{i j}(r, s)$.

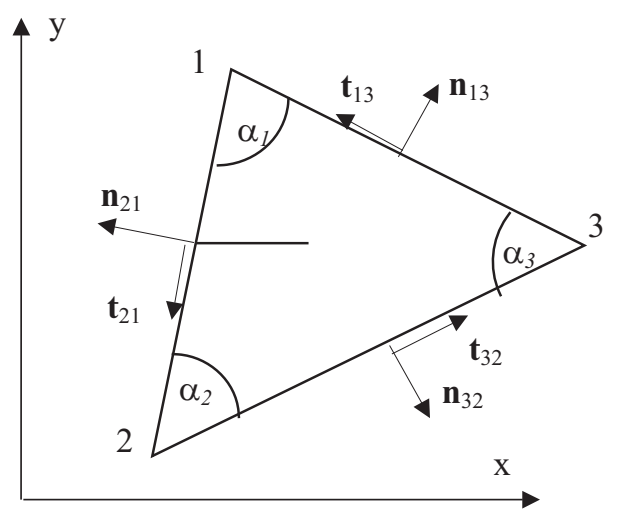

Fig. 4: Introducing geometrical quantity

The total displacements along the element sides in node $(r, s)$ due to the ${ }^{\Delta} u_{i j}$ (Eq. 1) and ${ }^{\varphi} u_{i j}$ (Eq. 3) may be expressed

$u_{i j}(r, s)=L_{i}{ }^{\Delta} u_{i j}-\varphi_{i} l_{j k} L_{k} L_{j}^{2} \sin \alpha_{i}-\varphi_{j} l_{j k} L_{k} L_{i}^{2} \sin \alpha_{j}$,

or more compactly

$$
\mathbf{u}^{*}(r, s)=\mathbf{N u}{ }^{*},
$$

where vector $\mathbf{u}^{*}(r, s)=\left\{u_{21}(r, s), u_{32}(r, s), u_{13}(r, s)\right\}^{T}$ represents the pure displacements of node $(r, s)$ in the directions of sides $i j, \mathbf{u}^{*}(r, s)=\left\{{ }^{\Delta} u_{21},{ }^{\Delta} u_{32},{ }^{\Delta} u_{13}, \varphi_{1}, \varphi_{2}, \varphi_{3}\right\}^{T}$ is the vector of six element conectors - elongations of the sides and pure node rotations, and $\mathbf{N}$ is the $(3 \times 6)$ matrix composed of the linear and cubic functions obtained directly from Eq. (4) for $i=2,3,1$ and $j=1,2,3$.

\section{Pure element stiffness matrix}

The individual components of the side strains are obtained from the pure displacement vector $\mathbf{u}^{*}(r, s)$ using the geometrical equations

$$
\varepsilon_{i j}=\frac{\mathrm{d} u_{i j}(r, s)}{\mathrm{d} s_{i j}}=\frac{\mathrm{d} u_{i j}(r, s)}{l_{i j} \mathrm{~d} L_{j}}-\frac{\mathrm{d} u_{i j}(r, s)}{l_{i j} \mathrm{~d} L_{i}} .
$$

Note that $\varepsilon_{i j}$ corresponds to a relative deformation along the side $i j$. Substituting Eq. (5) into Eq. (6) gives

$$
\varepsilon^{*}=\mathbf{B u}^{*} \text {, }
$$

where $\boldsymbol{\varepsilon}^{*}=\left\{\varepsilon_{21}, \varepsilon_{32}, \varepsilon_{13}\right\}^{T}$ and $\mathbf{B}$ is the element strain matrix with the components $B_{i k}=\frac{\partial N_{i k}}{\partial l_{i j}}(k=1, \ldots, 6)$.

Components of the engineering strain $\varepsilon=\left\{\varepsilon_{x}, \varepsilon_{y}, \gamma_{x y}\right\}^{T}$ are obtained applying standard transformation as

$\varepsilon_{i j}=\left\{\cos ^{2} \alpha_{i j}, \sin ^{2} \alpha_{i j}, \sin \alpha_{i j} \cos \alpha_{i j}\right\} \cdot \varepsilon$,

and in matrix notation

$$
\varepsilon^{*}=\mathbf{A} \cdot \varepsilon \Rightarrow \mathbf{A}^{-1} \varepsilon^{*}=\varepsilon .
$$

Substituting Eq. (7) into (8) finally provides

$$
\varepsilon=\mathbf{A}^{-1} \mathbf{B} \mathbf{u}^{*} \text {. }
$$

The finite element formulation used in this paper is based on the principle of minimum potential energy. The element stiffness matrix is calculated by considering the strain energy in the form

$$
\begin{aligned}
& E_{i(s)}=\frac{1}{2} \int_{V} \varepsilon^{T} \sigma \mathrm{d} V=\frac{1}{2} \mathbf{u}^{*} \int_{V} \mathbf{B}^{T} \mathbf{A}^{-T} \mathbf{D} \mathbf{A}^{-1} \mathbf{B} \mathrm{d} V, \\
& \mathbf{u}^{*} \Rightarrow \mathbf{K}^{*}=\frac{1}{2} \int_{V} \mathbf{B}^{T} \mathbf{A}^{-T} \mathbf{D} \mathbf{A}^{-1} \mathbf{B} \mathrm{d} V,
\end{aligned}
$$

where $\mathbf{D}$ is the elastic material stiffness matrix.

Seven Gauss integration points with $\mathfrak{R}\left(h^{5}\right)$ need to be used for numerical evalutions of $\mathbf{K}^{*}$, as the stiffness matrix contains functions of the fourth order.

\section{Element stiffness matrix}

The pure element stiffness matrix is extended by including the rigid body motions through the static matrix $\mathbf{T}$ obtained from the relations between the pure displacements

$$
\mathbf{u}^{*}=\left\{{ }^{\Delta} u_{21},{ }^{\Delta} u_{32},{ }^{\Delta} u_{13}, \varphi_{1}, \varphi_{2}, \varphi_{3}\right\}^{T}
$$

and the global node displacements

$$
\mathbf{u}=\left\{u_{1}, v_{1}, \Theta_{1}, u_{2}, v_{2}, \Theta_{2}, u_{3}, v_{3}, \Theta_{3}\right\}^{T} .
$$

Rigid body motions of the edges are decomposed into translations and rotations.

The relations between elongations of edges ${ }^{\Delta} u_{i j}$ and Cartesian displacements $u_{i}, v_{i}$ at the nodes are evident from Fig. 5

$$
\begin{aligned}
{ }^{\Delta} u_{i j}(r, s) & =\Delta u_{i j} \cdot \cos \alpha_{i j}+\Delta v_{i j} \cdot \sin \alpha_{i j}= \\
& =\left(u_{i}-u_{j}\right) \cos \alpha_{i j}+\left(v_{i}-v_{j}\right) \sin \alpha_{i j} .
\end{aligned}
$$




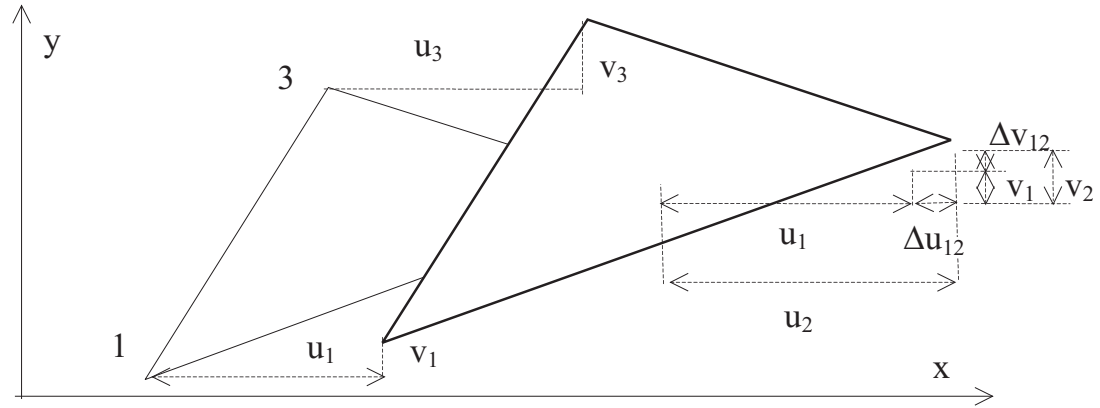

Fig. 5: Rigid body motions - translation

The relations among pure vertex rotations $\varphi_{i}$ and node rotations $\Theta_{i}$ shown in Fig. 6, are

$$
\Theta_{i}=\varphi_{i}+\bar{\Theta},
$$

where $\varphi_{i}$ is the pure rotation,

$\Theta_{i}$ represents the node rotation,

$\bar{\Theta}$ corresponds to a rigid body rotation given by

$\bar{\Theta}=\frac{1}{2}\left(-\frac{\partial u}{\partial y}+\frac{\partial v}{\partial x}\right)$.

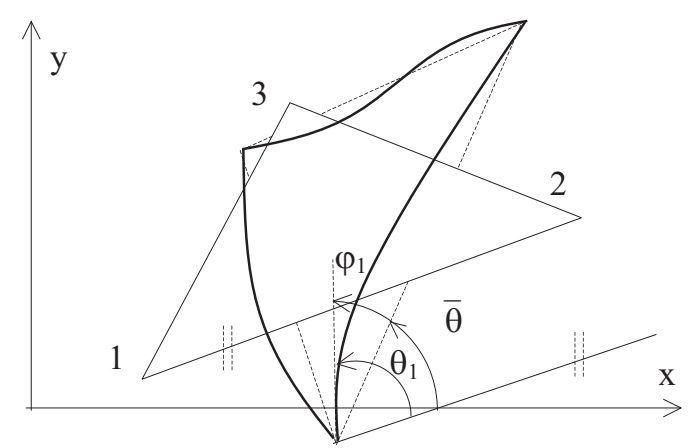

Fig. 6 Rigid body motions - rotation

The contribution of the pure rotations to the nodal displacements $u, v$ in Eq. (13) is neglected, since they cause no translations of the apex of a triangle. When using linear functions, the displacement field assumes the form.

$$
\begin{aligned}
u_{(r, s)} & =\sum_{i=1,3} L_{i} u_{i}, \quad v_{(r, s)}=\sum_{i=1,3} L_{i} v_{i}, \\
\bar{\Theta}_{i} & =\frac{1}{2}\left(-\sum_{i=1,3} \frac{\partial L_{i} u_{i}}{\partial y}+\sum_{i=1,3} \frac{\partial L_{i} v_{i}}{\partial x}\right)= \\
& =\frac{1}{4 A}\left(-\sum_{i=1,3} c_{i} u_{i}+\sum_{i=1,3} b_{i} v_{i}\right) .
\end{aligned}
$$

Combining Eqs. (12), (13), (14), provides the relation between pure displacements $\mathbf{u}^{*}$ and node displacements $\mathbf{u}$ in the form

$$
\mathbf{u}^{*}=\mathbf{T} \mathbf{u},
$$

where $\mathbf{u}=\left\{u_{1}, v_{1}, \Theta_{1}, u_{2}, v_{2}, \Theta_{2}, u_{3}, v_{3}, \Theta_{3}\right\}^{T}$ is the vector of nodal degrees of freedom $\left(u_{i}, v_{i}\right.$ are nodal Cartesian displacements and $\Theta_{i}$ are nodal rotations).

Note that the static matrix $\mathbf{T}$ is constant and does not cause any artifical strain or stress during the pure rigid body motions.

Relationship (15) is used in the expression for the strain energy (10), from which the element stiffness matrix $\mathbf{K}$ is obtained

$$
E_{i(s)}=\frac{1}{2} \mathbf{u}^{\mathrm{T}} \mathbf{T}^{\mathrm{T}} \mathbf{K}^{*} \mathbf{T} \mathbf{u} \Rightarrow \mathbf{K}=\mathbf{T}^{\mathrm{T}} \mathbf{K}^{*} \mathbf{T} .
$$

\subsection{Cantilever beam under tip load}

A standard test example often used in the literature corresponds to a shear-loaded cantilever beam. In particular a cantilever beam of rectangular cross section is subjected to parabolic traction at its tip with the resultant $F$ equal to $40 \mathrm{kN}$. The geometry and boundary conditions of the beam are shown in Fig. 7. The beam thickness is $1 \mathrm{~m}$. The following material properties are used: Young's modulus $E=30000 \mathrm{kPa}$ and Poisson's ratio $v=0.25$. The vertical deflection at point $c$ according to the theory of elasticity, ref. [6], is $w_{c}=0.3558 \mathrm{~m}$.

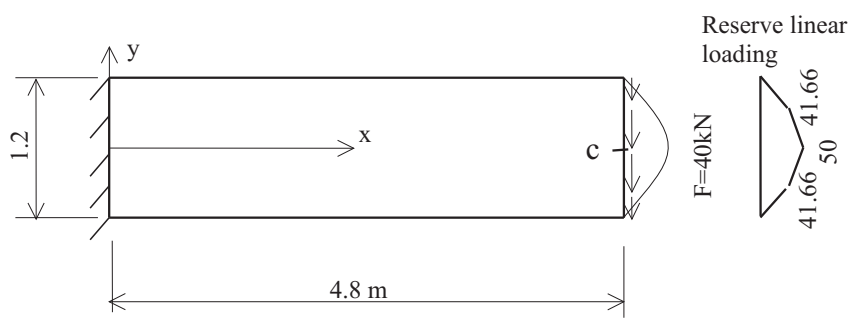

Fig. 7: Cantilever beam

Table 1 lists the numerical results obtained for the tip deflection. These are compared with the results obtained using the constant strain triangle (CST), the linear strain triangle (LST), the element of Allman [1] and Lee [5]. The values of the tip deflection obtained with the present element are closer to the exact solution than those obtained using the CST and the element due to Lee. The high accuracy of the results calculated here with a mesh of 128 elements is particularly noteworthy (even outperforming Allman's element). 
Table 1

\begin{tabular}{|c|c|c|c|c|l|}
\hline \multirow{2}{*}{$\begin{array}{c}\text { Number of } \\
\text { elements }\end{array}$} & \multicolumn{5}{|c|}{ Deflection $w_{c}(\mathrm{~m})$} \\
\cline { 2 - 6 } & CST & LST & Allman & Lee & Present \\
\hline 8 & 0.0909 & & 0.2696 & 0.2068 & 0.240 \\
\hline 32 & 0.1988 & 0.3550 & 0.3261 & 0.2958 & 0.3151 \\
\hline 128 & 0.3115 & 0.3556 & 0.3471 & 0.3388 & 0.3480 \\
\hline
\end{tabular}

The three adopted mesh patterns are shown in Fig. 8.

\subsection{Cantilever beam under end moment}

The next example is a cantilever beam subjected to the end moment $M=100$. The specimen dimensions together with the support and loading conditions are illustrated in Fig. 10. The modulus of elasticity and the Poisson ratio are set equal to $E=768, v=0.25$ so that the exact tip deflection is 100. The adopted mesh patterns ranging from $32 \times 2$ to $2 \times 2$ (Fig. 11) are used to examine the element aspect ratio ranging from $1: 1$ to $16: 1$.
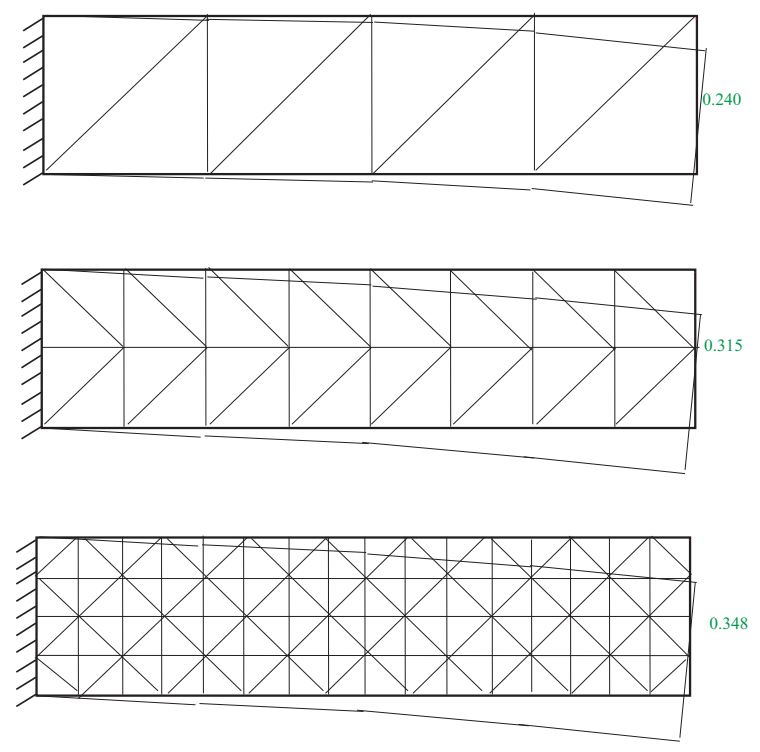

Fig. 8: Mesh patterns

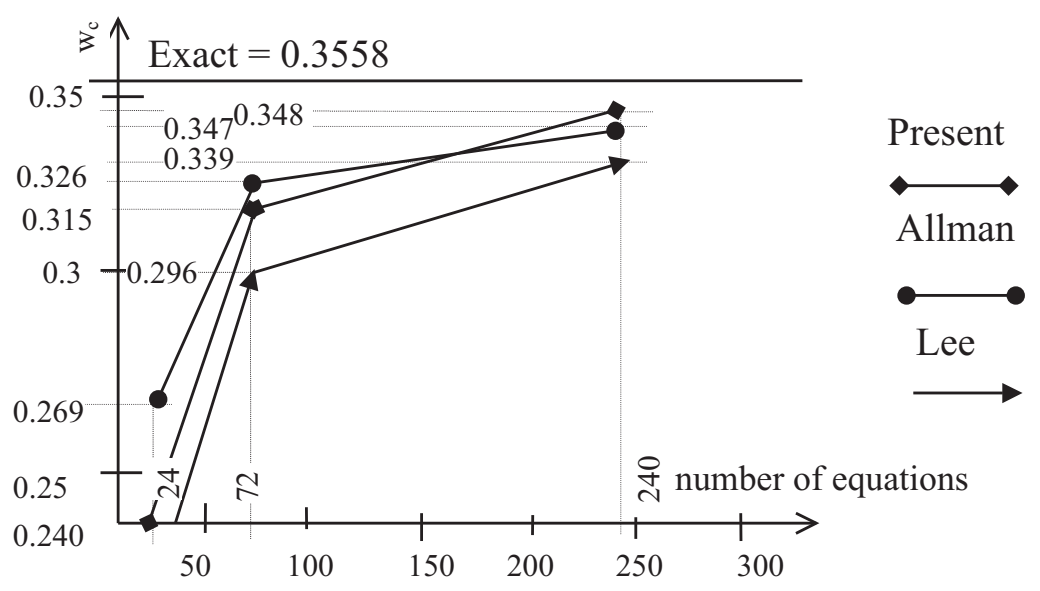

Fig. 9: Dependence of the deflection $w_{c}$ on the number of equations

Table 2: Tip deflections for a beam under end moment

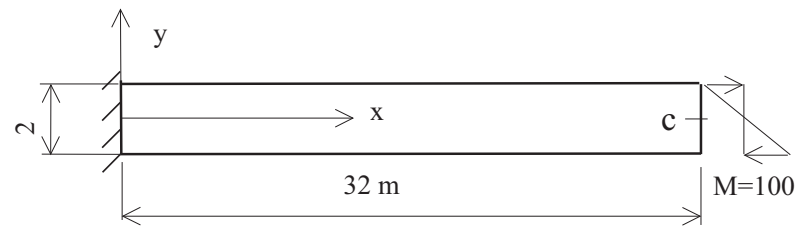

Fig. 10: Cantilever beam

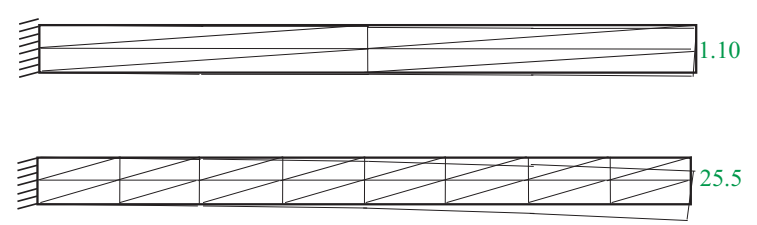

Fig. 11: Mesh patterns $2 \times 2,8 \times 2$

\begin{tabular}{|c|c|c|c|c|c|}
\hline \multirow{2}{*}{$\begin{array}{c}\text { Number of } \\
\text { elements }\end{array}$} & \multicolumn{5}{|c|}{ Deflection $w_{c}(\mathrm{~m})$} \\
\cline { 2 - 6 } & CST & EFFAND & Allman 3i & $\begin{array}{c}\text { Allman 7 } \\
\mathrm{i}\end{array}$ & Present \\
\hline $2 \times 2$ & 1.28 & 100.07 & 0.39 & 0.16 & 1.10 \\
\hline $4 \times 2$ & 4.82 & 99.96 & 5.42 & 2.47 & 3.31 \\
\hline $8 \times 2$ & 15.75 & 99.99 & 38.32 & 24.25 & 25.53 \\
\hline $16 \times 2$ & 36.36 & 99.99 & 76.48 & 69.09 & 69.70 \\
\hline
\end{tabular}

This element performs well for unit aspect ratios, but rapidly becomes worse for aspect ratios over 2:1. The results for the present element are compared in Fig. 12 with the results obtained using the constant strain triangle (CST), the element of Allman [1] and the EFFAND element due to Felippa and Alexander [2, 3]. Numerical results for the tip deflexion $w_{c}$ at point $\mathrm{c}$ are also given in Table 2 . 


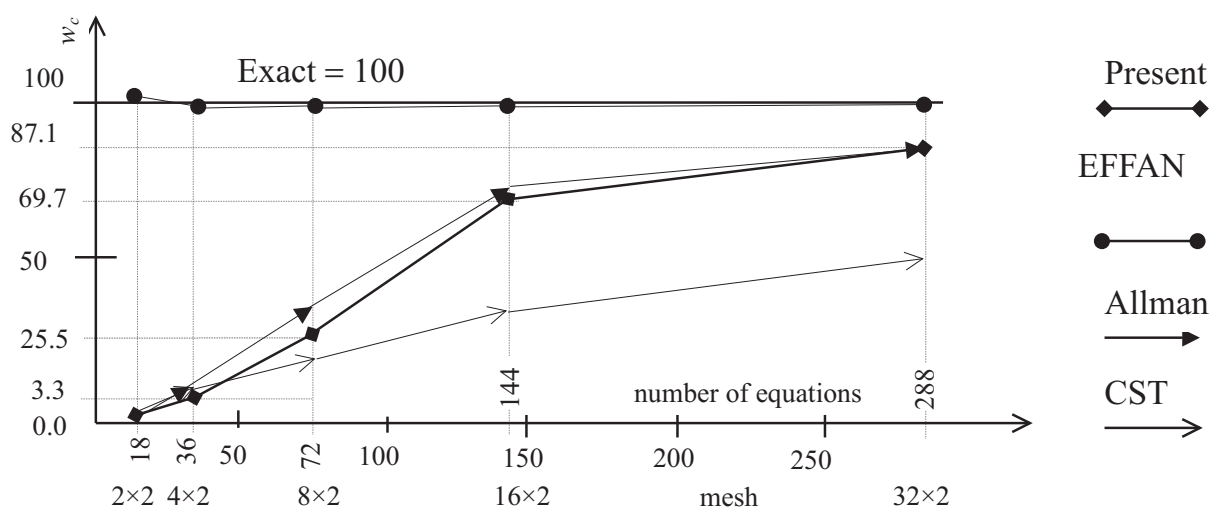

Fig. 12: Dependence of deflection $w_{c}$ and number of equations

\section{Conclusions}

This paper presents the derivation of a simple triangular membrane finite element using the principle of minimum potential energy. The formulation of two stiffness matrices the pure stiffness matrix depending on elongations of the edges and the global matrix depending on nodal displacements $u_{i}, v_{i}, \varphi_{i}$ - enables nonlinear problems to be solved more effectively and quickly due to the simple calculation of the unbalanced forces. It is advantage in comparison with quadrilateral elements [4].

The element can represent exactly all constant strain states. The numerical results from the selected test example show that quite acceptable accuracy is achieved for practical applications.

\section{Appendix A}

\section{Area Coordinates}

The use of area coordinates enables the relation for one edge or one vertex to be derived. The other relations are obtained by cyclic substitution $i$ from 1 to 2 , from 2 to 3 , from 3 to 1 .

$$
L_{i}=\frac{A_{i}}{A}=\frac{\left(a_{i}+b_{i} x+c_{i} y\right)}{2 A},
$$

where $A$ is the area of the triangle,

$$
a_{i}=x_{j} y_{k}-x_{k} y_{j}, \quad b_{i}=y_{j}-y_{k}, \quad c_{i}=x_{j}-x_{k},
$$

and $x_{i}, y_{i}$ are the coordinates of the node in Fig.13.

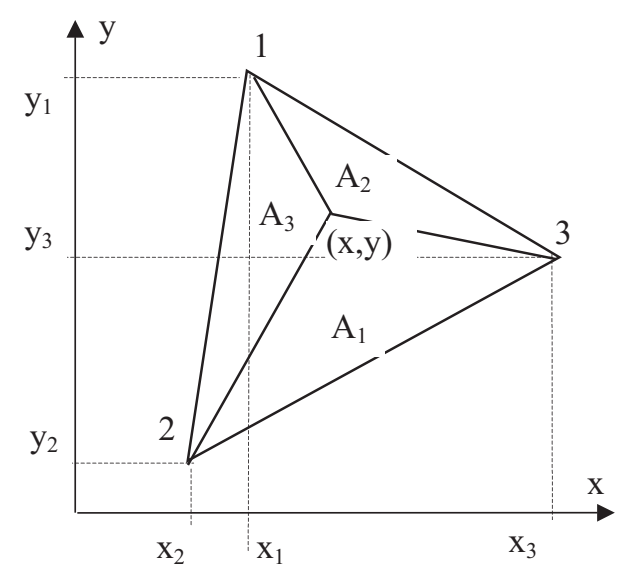

Fig. 13: Area coordinates

\section{Acknowledgment}

Financial support from GAČR 103020658 and MSM 210000001 is gratefully acknowledged.

\section{References}

[1] Allman D. J: "A Compatible triangular Element including vertex rigid rotations for Plane elasticity, Analysis." Computer \& structures, Vol. 19 (1984).

[2] Bergan P. G., Felippa C. A.: "A triangular membrane element with rotational degrees of freedom." Computer methods in app. Mech. and Eng., Vol. 50 (1985), p. 25-69.

[3] Felippa C. A., Alexander S.: "Membrane triangles with corner drilling freedoms - III. Implementation and performance evalution." Finite Elements in Analysis and Design, Vol. 12 (1992), p. 203-239.

[4] Ibrahimbegovic A., Taylor R. L., Wilson E. L.: "A robust quadrilateral membrane finite element with drilling degrees of freedom." Int. Journal for numerical meth. In. Eng., Vol. 30 (1990), p. 445-457.

[5] Lee S. C., Yoo CH. H.: "A Novel Shell element including in plane Torque effect." Computer \& structures, Vol. 28 (1988).

[6] Timoshenko S., Goodier J. N.: "Theory of elasticity." $3^{\text {rd }}$ Edn., McGraw-Hill, New York, 1970.

Petr Fajman

phone: +420224354473

e-mail: fajman@fsv.cvut.cz

Department of Structural Mechanics

Czech Technical University

Faculty of Civil Engineering

Thákurova 7

16629 Praha 6, Czech Republic 